\title{
Effects of attenuation map accuracy on attenuation-corrected micro-SPECT images
}

\author{
Chao $\mathrm{Wu}^{1,2^{*}}$, Hugo A Gratama van Andel ${ }^{2,3}$, Peter Laverman ${ }^{4}$, Otto C Boerman ${ }^{4}$ and Freek J Beekman ${ }^{1,3}$
}

\begin{abstract}
Background: In single-photon emission computed tomography (SPECT), attenuation of photon flux in tissue affects quantitative accuracy of reconstructed images. Attenuation maps derived from X-ray computed tomography (CT) can be employed for attenuation correction. The attenuation coefficients as well as registration accuracy between SPECT and CT can be influenced by several factors. Here we investigate how such inaccuracies influence micro-SPECT quantification.
\end{abstract}

Methods: Effects of (1) misalignments between micro-SPECT and micro-CT through shifts and rotation, (2) globally altered attenuation coefficients and (3) combinations of these were evaluated. Tests were performed with a NEMA NU 4-2008 phantom and with rat cadavers containing sources with known activity.

Results: Changes in measured activities within volumes of interest in phantom images ranged from $<1.5 \%\left({ }^{125} \mathrm{l}\right)$ and $<0.6 \%\left({ }^{201} \mathrm{TI},{ }^{99 \mathrm{~m}} \mathrm{Tc}\right.$ and $\left.{ }^{111} \mathrm{In}\right)$ for 1 -mm shifts to $<4.5 \%\left({ }^{125} \mathrm{I}\right)$ and $<1.7 \%\left({ }^{201} \mathrm{Tl},{ }^{99 \mathrm{~m}} \mathrm{Tc}\right.$ and $\left.{ }^{111} \mathrm{In}\right)$ with large misregistration $\left(3 \mathrm{~mm}\right.$ ). Changes induced by $15^{\circ}$ rotation were smaller than those by $3-\mathrm{mm}$ shifts. By significantly altering attenuation coefficients $( \pm 10 \%)$, activity changes of $<5.2 \%$ for ${ }^{125} \mathrm{I}$ and $<2.7 \%$ for ${ }^{201} \mathrm{Tl},{ }^{99 \mathrm{~m}} \mathrm{Tc}$ and ${ }^{111} \mathrm{In}$ were induced. Similar trends were seen in rat studies.

Conclusions: While getting sufficient accuracy of attenuation maps in clinical imaging is highly challenging, our results indicate that micro-SPECT quantification is quite robust to various imperfections of attenuation maps.

Keywords: Molecular imaging, SPECT, CT, Small animal, Misregistration, Attenuation correction, Quantification

\section{Background}

Small-animal single-photon emission computed tomography (micro-SPECT) plays an increasingly important role in biomedical research [1-9]. Attenuation correction, together with correction for effects such as scatter and camera blurring, is essential for obtaining highly quantitative SPECT images. Efforts have been made to improve quantitative accuracy in micro-SPECT. For instance, Hwang et al. [10] and Vanhove et al. [11] showed that by using attenuation maps derived from micro-CT data, cupping artefacts in micro-SPECT images could be eliminated and quantitative errors could be reduced with iterative attenuation correction [12] and window-based scatter correction [13] methods. We recently introduced

\footnotetext{
* Correspondence: c.wu@tudelft.nl

'Section Radiation, Detection \& Medical Imaging, Delft University of Technology, Mekelweg 15, Delft 2629 JB, the Netherlands

${ }^{2}$ Rudolf Magnus Institute of Neuroscience, University Medical Center Utrecht, STR 5.101, Univeriteitsweg 100, Utrecht 3584 CG, the Netherlands Full list of author information is available at the end of the article
}

an optical-contour-based modified Chang method [14] for attenuation correction in multi-pinhole SPECT, which is applied post-reconstruction and leads to small quantitative errors $(1.7 \%$ and $2.1 \%$ on average in phantom and rat studies with ${ }^{99 \mathrm{~m}} \mathrm{Tc}$, respectively) [15]. This method was later extended to a CT-based non-uniform attenuation correction and tested with four different isotopes on a micro-SPECT/CT system [16]. Average quantitative errors of $2.1 \%, 3.3 \%, 2.0 \%$ and $2.0 \%$ in activities of sources implanted in rat cadavers were achieved for ${ }^{125} \mathrm{I},{ }^{201} \mathrm{Tl},{ }^{99 \mathrm{~m}} \mathrm{Tc}$ and ${ }^{111} \mathrm{In}$, respectively.

In clinical SPECT, problems caused by inaccurate attenuation maps have been thoroughly investigated. For example, Tonge et al. $[17,18]$ indicated that even minor degrees of misregistration (a couple of millimetres) between SPECT and CT can create significant artefacts. Goetze et al. [19] found that misregistration of less than $1 \mathrm{~cm}$ contributed significantly to changes in myocardial perfusion images. Studies of e.g. Kennedy et al. [20]

\section{实}

(c) 2013 Wu et al.; licensee Springer. This is an Open Access article distributed under the terms of the Creative Commons Attribution License (http://creativecommons.org/licenses/by/2.0), which permits unrestricted use, distribution, and reproduction in any medium, provided the original work is properly cited. 
found misregistration of more than $3.4 \mathrm{~mm}$ in $73 \%$ of clinical SPECT/CT studies. Such studies even raised the question whether attenuation correction should be applied to clinical myocardial perfusion SPECT. Results about attenuation map inaccuracies and their effects on quantitative accuracy for clinical SPECT cannot be directly translated to micro-SPECT studies because of e.g. higher resolution, different anatomies and less attenuation in micro-SPECT studies. Since anatomical images from external scanners are often combined with microSPECT, registration and scaling issues occur quite often. Therefore, knowledge about required attenuation map accuracy is highly important.

The aim of the present study is to investigate the effects of attenuation map imperfections on the accuracy of micro-SPECT quantification. Two main sources of attenuation map inaccuracies were emulated: (1) misalignments between micro-SPECT and micro-CT images, e.g. as could result from misregistration or animal movement; and (2) global errors in the attenuation coefficients derived from micro-CT data, e.g. as could result from sub-optimal CT calibration.

\section{Methods}

Animal studies involved in this study were conducted following the regulations approved by the Animal Experiment Committee of the Radboud University Nijmegen.

\section{Phantom and animal imaging in U-SPECT-II/CT}

The U-SPECT-II/CT system used in the present study (MILabs, Utrecht, the Netherlands; Figure 1) comprises three stationary gamma cameras equipped with different cylindrical multi-pinhole collimators optimized for differently sized rodents, each containing 75 focused pinholes. An XYZ stage shifts the animal during acquisition for scanning large volumes up to the entire animal. The integrated high-throughput micro-CT comprises a fast circular cone-beam setup dedicated for low-dose scanning with an air-cooled metallo-ceramic tube (voltage range 20 to $65 \mathrm{kV}$ ) and a $1280 \times 1024$ pixel 12-bit CCDbased detector. Performance of this system is described in $[15,16,21-25]$.

A NEMA image quality phantom for small-animal imaging (NEMA NU 4-2008, NEMA, Rosslyn, VA, USA; Figure 2a) with two small air-filled chambers was first filled with ${ }^{99 \mathrm{~m}_{\mathrm{TcO}}} \mathrm{Tc}_{4}$ solution $(8.65 \mathrm{MBq} / \mathrm{ml})$. The activity was measured in a dose calibrator. Next, SPECT and fully circular CT $(60 \mathrm{kV}$ and $615 \mu \mathrm{A})$ were performed. Then, the same procedure was repeated for ${ }^{201} \mathrm{TlCl}_{3},{ }^{111} \mathrm{InCl}_{3}$ and $\mathrm{Na}^{125} \mathrm{I}$ solutions, with activity concentrations of $5.90 \mathrm{MBq} / \mathrm{ml}, 5.91 \mathrm{MBq} / \mathrm{ml}$ and $4.54 \mathrm{MBq} / \mathrm{ml}$, respectively. In addition, 12 small ${ }^{99 \mathrm{~m}} \mathrm{Tc}$ sources (approximately conical, about $6 \mathrm{~mm}$ in length and $3 \mathrm{~mm}$ in average diameter, and made from tips cut from $0.5-\mathrm{ml}$ microcentrifuge tubes; Figure $2 \mathrm{~b}$ ) with known activity were inserted into a cadaver of a female Wistar rat surgically (inside or near the mouth, neck, left and right lungs, liver, stomach, left kidney, intestine, left and right shoulders, and left and right sides of the waist, as shown in Figure 2c). Total-body SPECT/CT was performed for the rat. This was repeated with three other rat cadavers for ${ }^{125} \mathrm{I},{ }^{201} \mathrm{Tl}$ and ${ }^{111} \mathrm{In}$. Activities of the ${ }^{125} \mathrm{I},{ }^{201} \mathrm{Tl},{ }^{99 \mathrm{~m}} \mathrm{Tc}$ and ${ }^{111} \mathrm{In}$ sources ranged from 8.30 to $11.8 \mathrm{MBq}, 3.82$ to $7.23 \mathrm{MBq}, 13.9$ to $18.8 \mathrm{MBq}$ and 6.02 to $11.7 \mathrm{MBq}$, respectively.

SPECT images were reconstructed by using the scanning focus method [26] combined with pixel-based ordered subset expectation maximization [27] using 16 subsets, 6 iterations and a $0.375-\mathrm{mm}$ voxel grid, during which blurring and pinhole sensitivity effects were corrected [28] and an energy-window-based scatter correction method was implemented [13]. CT images were reconstructed with a $0.169-\mathrm{mm}$ voxel grid, using NRecon

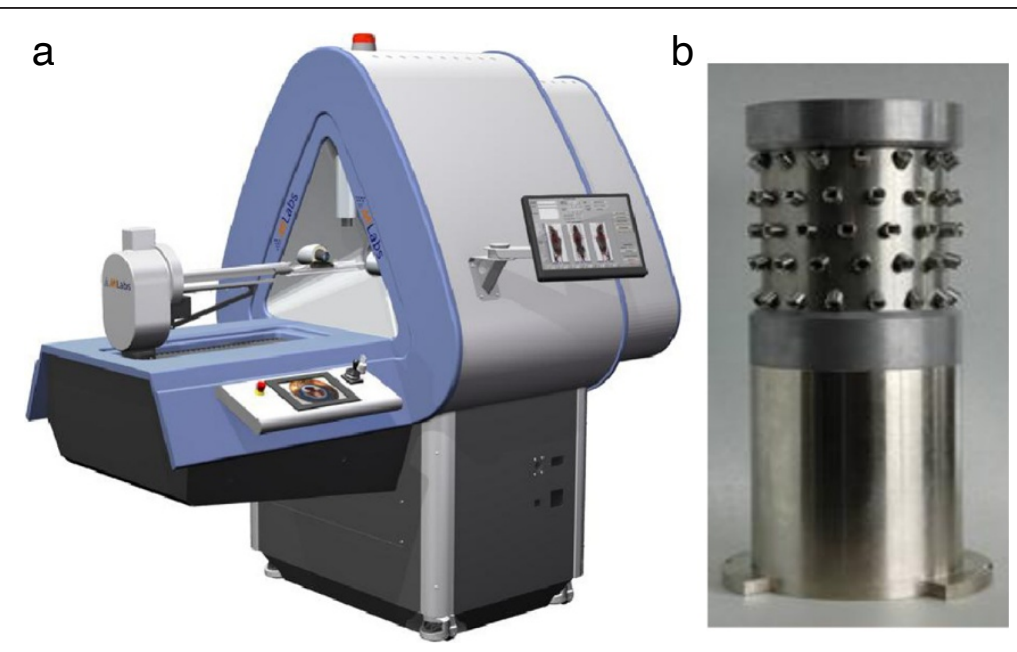

Figure 1 Images of the system and rat collimator used in the present study. (a) U-SPECT-II/CT multi-pinhole system and (b) rat collimator. 

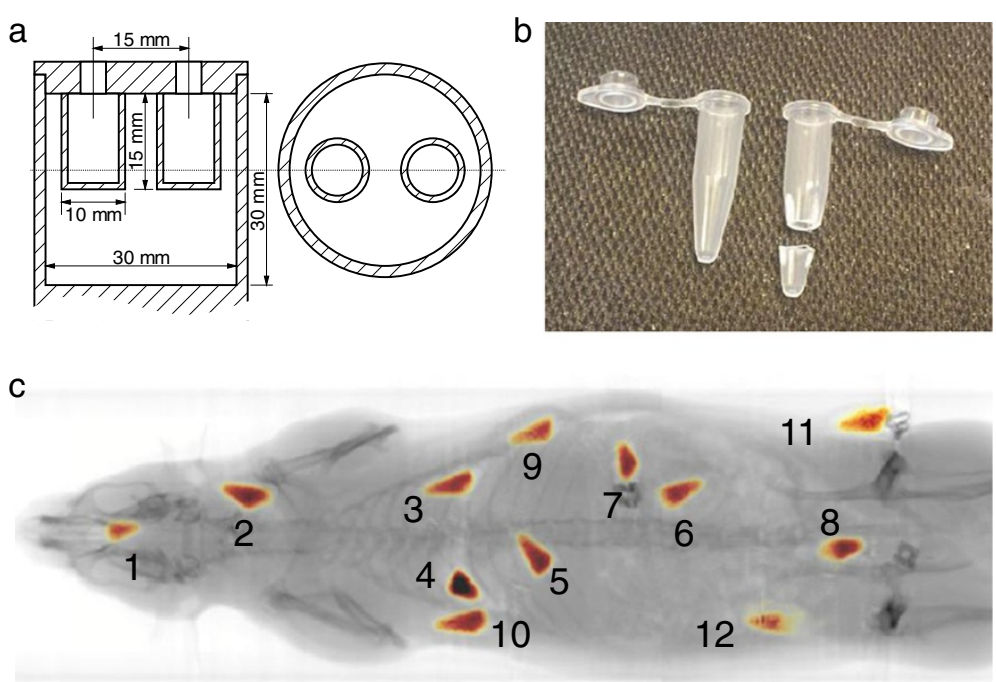

Figure 2 NEMA image quality phantom, microcentrifuge tube and rat body showing ${ }^{99 m}$ Tc sources. (a) Drawing of NEMA image quality phantom, (b) $0.5-\mathrm{ml}$ microcentrifuge tube and its tip for making sources (sealed with Parafilm (Pechiney Plastic Packaging Company, Chicago, IL, USA) afterwards) used in the rat study and (c) rat with numbered ${ }^{99 m}$ Tc sources with known activity.

(SkyScan, Kontich, Belgium) with its built-in modified Feldkamp algorithm and methods for beam hardening and ring artefact corrections. Both SPECT and CT images were rescaled with pre-measured calibration factors, and then SPECT images were registered to CT images by means of rigid transformations with pre-determined matrices.

More details on system calibration, phantom and animal preparation, data acquisition, image reconstruction, scatter correction and SPECT-CT registration have been described in [16].

\section{CT-based non-uniform attenuation correction for micro- SPECT}

The Chang algorithm [14] is a practical attenuation correction method, which provides an approximation for obtaining the transmitted fraction (TF) for each voxel in reconstructed images. Being a post-reconstruction correction method, the Chang algorithm does not require generation of additional SPECT system matrices that each takes up tremendous amounts of memory and computation. Therefore, the Chang algorithm can be easily applied to images acquired from any microSPECT system. This algorithm has been shown to lead to highly accurate results $[15,16]$ which can be attributed to the fact that (in contrast to clinical SPECT) only small corrections are required in rodents, justifying the use of this first-order method (in clinical SPECT, iterative attenuation correction is highly preferred while memory demands are different). Details about implementation of the modified Chang algorithm for multi-pinhole SPECT are found in [16]. In brief, the TF along every projection line from a certain voxel is calculated, and the average of all these TFs is treated as the overall TF for that voxel:

$$
\mathrm{TF}=\frac{1}{M} \sum_{m=1}^{M} \exp \left(-\int_{L_{m}} \mu(l) d l\right),
$$

where $M$ is the number of projections involved in acquisition for data of a certain voxel, $L_{m}$ denotes the $m$ th projection path of gamma photons and $\mu$ is the attenuation coefficient on that projection line $L_{m}$. Attenuation coefficients were derived from CT images by linear scaling [29]:

$$
\mu=\mu_{0}\left(\frac{\mathrm{HU}}{1,000}+1\right)
$$

in which $\mathrm{HU}$ is the CT image intensity in Hounsfield units, and $\mu_{0}$ is the linear attenuation coefficient associated with water and the energy of the photons used in SPECT. $\mu_{0}$ was obtained using the NIST data tables [30]. In case that more than one photon energy was involved, $\mu_{0}$ was chosen to be the average of $\mu_{0}(E)$ weighted by the number of photons detected under each energy $E$. When the TF for each voxel was estimated, the attenuation was compensated by dividing each voxel value by its corresponding TF [16].

\section{Attenuation correction with different attenuation maps}

For each scan, an attenuation coefficient map was created from the registered $\mathrm{CT}$ image by linear scaling as in Equation 2 and then converted to a transmitted fraction map with the non-uniform Chang algorithm as 
described in Equation 1. To only evaluate the impact of inaccuracies in attenuation maps on the SPECT quantification, we considered these original attenuation maps to be accurate, and thus, we used the SPECT images corrected with these maps as reference images. Meanwhile, quantitative errors, i.e. differences between activities measured in images and in a dose calibrator, were also calculated for each reference image for reasons of comparison.

To investigate the effect of misregistration, we shifted the original TF maps for phantom studies in either $+x$ (towards the right side of the system) or $+y$ (downwards) direction and the maps for animal studies in $+x,+y$ or + $z$ (towards the back side of the system) direction. The distances of shifting in each direction were set to $1 \mathrm{~mm}$ and $3 \mathrm{~mm}$. All original TF maps were also rotated anticlockwise by $15^{\circ}$ to emulate the consequences of animal movement during scans. All these shifted and rotated TF maps were used for attenuation correction of their corresponding SPECT images. Later on, we globally changed the attenuation coefficients in the attenuation maps by $\pm 10 \%$ of the original values. This was to emulate errors introduced by $\mathrm{CT}$ itself and/or from converting $\mathrm{CT}$ values to attenuation coefficients. TF maps were again calculated with the non-uniform Chang algorithm from these altered attenuation maps and then used for correcting for attenuation in their corresponding SPECT images. As a final test, the TF maps calculated with the $+10 \%$ attenuation coefficient maps were also rotated anticlockwise by $15^{\circ}$ and shifted by $3 \mathrm{~mm}$ in the $x$ direction. This way, we introduced a combination of 'worstcase' errors. These TF maps were then employed for attenuation correction. All images that were corrected with the abovementioned inaccurate attenuation maps were compared to the reference images.

All the above operations were performed for ${ }^{125} \mathrm{I}$, ${ }^{201} \mathrm{Tl},{ }^{99 \mathrm{~m}} \mathrm{Tc}$ and ${ }^{111}$ In separately.

\section{Results}

\section{Phantom experiments}

Three $3 \mathrm{~mm} \times 3 \mathrm{~mm} \times 11 \mathrm{~mm}$ volumes of interest (VOIs) were defined in heterogeneous slices of each phantom image, as shown in Figure 3a. Measured activities within the VOIs in the reference images and in the images corrected with inaccurate attenuation maps were calculated and compared. Changes in the measured VOI activities induced by attenuation map inaccuracies are listed in Table 1. With $1-\mathrm{mm}$ shifts of the attenuation maps, the VOI activity changed less than $1.5 \%$ for ${ }^{125} \mathrm{I}$ and $0.6 \%$ for ${ }^{201} \mathrm{Tl},{ }^{99 \mathrm{~m}} \mathrm{Tc}$ and ${ }^{111} \mathrm{In}$. When the shifts increased to $3 \mathrm{~mm}$, the changes increased to less than $4.5 \%$ for ${ }^{125} \mathrm{I}$ and $1.7 \%$ for ${ }^{201} \mathrm{Tl},{ }^{99 \mathrm{~m}} \mathrm{Tc}$ and ${ }^{111} \mathrm{In}$. With $15^{\circ}$ rotation of the maps, the changes were less than $1.4 \%$ for ${ }^{125} \mathrm{I}$ and $0.9 \%$ for ${ }^{201} \mathrm{Tl},{ }^{99 \mathrm{~m}} \mathrm{Tc}$ and ${ }^{111} \mathrm{In}$. When the attenuation coefficients were altered by $10 \%$, the activity in the VOIs changed less than $5.2 \%$ for ${ }^{125} \mathrm{I}$ and $2.7 \%$ for ${ }^{201} \mathrm{Tl},{ }^{99 \mathrm{~m}} \mathrm{Tc}$ and ${ }^{111} \mathrm{In}$. With the combination of rotation and shifts of the maps and altered attenuation coefficients, the VOI activity changed less than $6.2 \%$ for ${ }^{125} \mathrm{I}$ and $3.0 \%$ for ${ }^{201} \mathrm{Tl},{ }^{99 \mathrm{~m}} \mathrm{Tc}$ and ${ }^{111} \mathrm{In}$. The quantitative errors of the reference images are listed in the first data column of Table 1 for reasons of comparison.

In addition, the normalized root mean square deviation (NRMSD) was calculated voxel-wise between the reference images and the images corrected with inaccurate attenuation maps:

$$
\mathrm{NRMSD}=\sqrt{\frac{1}{n} \sum_{i=1}^{n}\left(\frac{v_{i}-v r_{i}}{v r_{i}}\right)^{2}} \times 100 \%
$$

where $v_{i}$ is $i$ th voxel inside the phantom chamber in an image corrected with an inaccurate map, and $v r_{i}$ is the corresponding voxel in a reference image. $n$ is the number of voxels inside the phantom chamber. The results are listed in Table 2. The largest NRMSDs caused by combined rotation, shifts and attenuation coefficient errors were $6.3 \%, 3.0 \%, 2.5 \%$ and $2.3 \%$ for ${ }^{125} \mathrm{I},{ }^{201} \mathrm{Tl}$, ${ }^{99 \mathrm{~m}} \mathrm{Tc}$ and ${ }^{111} \mathrm{In}$, respectively.

Line profiles through homogenous and heterogeneous slices (defined in Figure 3a) were created from images corrected with different attenuation maps and are plotted in Figure 3b. When the attenuation map was shifted, it is clear that the main changes in quantitation were located at the edges of the phantom along the shifting direction. The changes were small for ${ }^{201} \mathrm{Tl},{ }^{99 \mathrm{~m}} \mathrm{Tc}$ and ${ }^{111}$ In imaging $(\leq 5 \%)$, while for ${ }^{125} \mathrm{I}$ it was larger $(\leq 10 \%)$. Rotation of attenuation maps had almost no effect in homogenous slices for all four isotopes: the line profiles perfectly overlapped the reference profiles. In heterogeneous slices, the profiles slightly deviated ( $1 \%$ to $2 \%$ ) from the reference profiles only close to the edges of the air chambers. When altering the attenuation coefficients by $\pm 10 \%$, the induced changes were on average about $2 \%$ to $3 \%$ for ${ }^{201} \mathrm{Tl},{ }^{99 \mathrm{~m}} \mathrm{Tc}$ and ${ }^{111} \mathrm{In}$, and about $5 \%$ for ${ }^{125} \mathrm{I}$. Unlike shifting or rotating the maps, the changes in quantitation when the attenuation coefficients were altered resulted in global (but not uniform) over- or underestimations.

\section{Animal experiments}

In the rat SPECT images, non-overlapped spherical VOIs (diameter of about $15 \mathrm{~mm}$ ) were created for each small source. The sources were completely enclosed in the centres of their corresponding VOIs. Activities in the VOIs were measured in the images corrected with different attenuation maps. Comparisons were made between measurements in the reference images and in the images corrected with inaccurate maps. Changes in the 
a
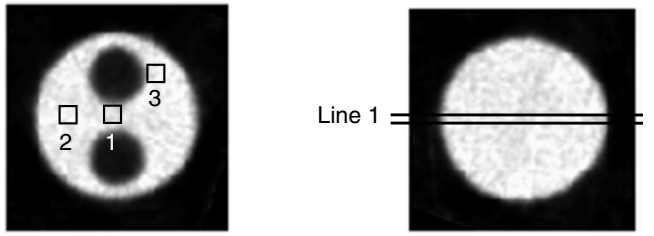

Line 1

b
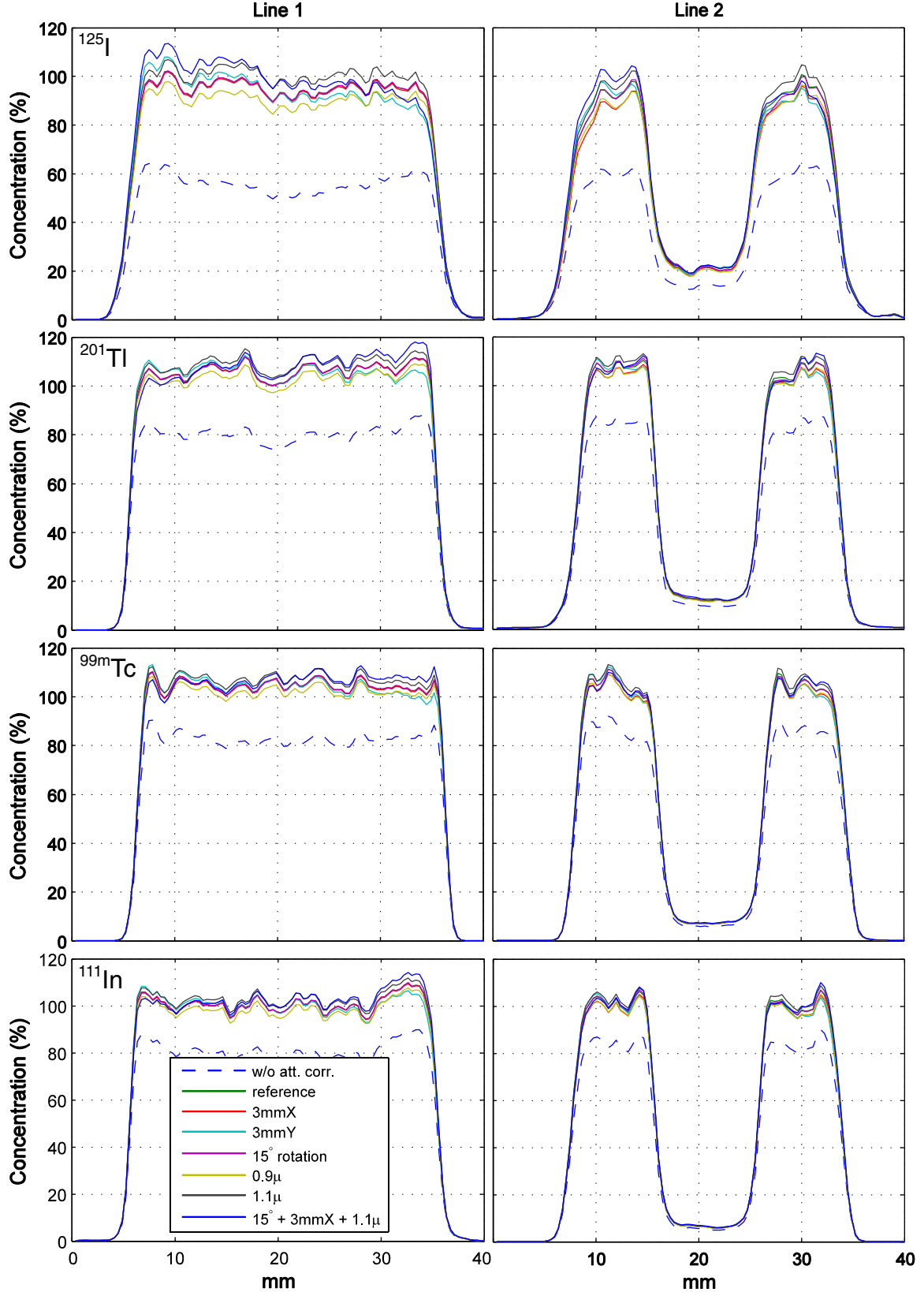

Figure 3 VOIs and line profiles. (a) VOI and line-profile definitions in phantom images. (b) Line profiles in phantom images with different attenuation map inaccuracies for four isotopes. 
Table 1 Quantitative errors and relative changes of VOI activities

\begin{tabular}{|c|c|c|c|c|c|c|c|c|c|c|}
\hline \multirow[t]{4}{*}{ Isotope } & \multirow[t]{4}{*}{ VOI } & \multirow{4}{*}{$\begin{array}{c}\text { Quantitative } \\
\text { error }\end{array}$} & \multicolumn{8}{|c|}{ Changes induced by map inaccuracies (rotation, shift and/or $\mu$ change) with respect to references } \\
\hline & & & $0^{\circ}$ & $0^{\circ}$ & $0^{\circ}$ & $0^{\circ}$ & $15^{\circ}$ & $0^{\circ}$ & $0^{\circ}$ & $15^{\circ}$ \\
\hline & & & $1 \mathrm{~mm} X$ & $3 \mathrm{~mm} X$ & $1 \mathrm{~mm} Y$ & $3 \mathrm{~mm} Y$ & $0 \mathrm{~mm}$ & $0 \mathrm{~mm}$ & $0 \mathrm{~mm}$ & $3 \mathrm{~mm} X$ \\
\hline & & & $1.0 \mu$ & $1.0 \mu$ & $1.0 \mu$ & $1.0 \mu$ & $1.0 \mu$ & $0.9 \mu$ & $1.1 \mu$ & $1.1 \mu$ \\
\hline \multirow[t]{3}{*}{${ }^{125}$} & 1 & -20.4 & 0.1 & 0.4 & -0.5 & -4.0 & 0.0 & -4.9 & 5.2 & 5.3 \\
\hline & 2 & -11.8 & 0.7 & 1.1 & -0.2 & -1.0 & -0.2 & -4.8 & 5.0 & 6.2 \\
\hline & 3 & -7.9 & -1.1 & -4.0 & -1.5 & -4.5 & -1.4 & -4.2 & 4.3 & -0.8 \\
\hline \multirow[t]{3}{*}{${ }^{201} \mathrm{Tl}$} & 1 & -5.8 & 0.0 & 0.1 & -0.1 & -1.5 & 0.0 & -2.6 & 2.7 & 2.7 \\
\hline & 2 & 6.1 & -0.4 & -1.4 & 0.0 & -0.3 & -0.1 & -2.5 & 2.6 & 3.0 \\
\hline & 3 & 4.4 & 0.2 & -0.3 & 0.6 & 1.4 & -0.7 & -2.3 & 2.3 & 0.0 \\
\hline \multirow[t]{3}{*}{${ }^{99 \mathrm{~m}} \mathrm{Tc}$} & 1 & -2.0 & 0.1 & 0.2 & -0.2 & -1.7 & 0.0 & -2.2 & 2.2 & 2.1 \\
\hline & 2 & 6.1 & -0.3 & -1.1 & -0.1 & -0.3 & -0.1 & -2.1 & 2.2 & 2.4 \\
\hline & 3 & -8.3 & 0.4 & -0.2 & 0.5 & 1.3 & -0.2 & -1.8 & 1.9 & 0.2 \\
\hline \multirow[t]{3}{*}{${ }^{111} \operatorname{In}$} & 1 & -4.7 & 0.0 & 0.1 & -0.2 & -1.4 & 0.0 & -2.1 & 2.1 & 2.2 \\
\hline & 2 & -3.5 & -0.4 & -1.2 & 0.0 & -0.3 & -0.1 & -2.0 & 2.0 & 2.5 \\
\hline & 3 & 3.2 & 0.0 & -1.1 & 0.4 & 0.9 & -0.9 & -1.9 & 1.9 & 0.6 \\
\hline
\end{tabular}

Quantitative errors (in \%) of VOI activities in reference phantom SPECT images were calculated with respect to dose calibrator measurements (1st data column), and relative changes (in \%) in VOI activities due to attenuation map inaccuracies were calculated with respect to reference images (2nd to 9 th data column).

measured VOI activities induced by map inaccuracies are plotted in Figure 4, and the average absolute changes in percentage are listed in Table 3. With 1-mm shifts of the maps, the measured VOI activities changed $1.4 \pm$ $1.1 \%, 0.6 \pm 0.5 \%, 0.5 \pm 0.4 \%$ and $0.5 \pm 0.4 \%$ on average for ${ }^{125} \mathrm{I},{ }^{201} \mathrm{Tl},{ }^{99 \mathrm{~m}} \mathrm{Tc}$ and ${ }^{111} \mathrm{In}$, respectively, while these average changes increased to $3.6 \pm 2.9 \%, 1.5 \pm 1.2 \%, 1.3 \pm$ $1.2 \%$ and $1.2 \pm 1.0 \%$ with 3 -mm shifts. When the maps were rotated by $15^{\circ}$, the changes were $2.1 \pm 1.8 \%, 0.8 \pm$ $0.8 \%, 0.5 \pm 0.4 \%$ and $0.5 \pm 0.8 \%$ on average. When altering the attenuation coefficients by $10 \%$, the VOI activities changed $5.9 \pm 1.8 \%, 3.3 \pm 0.9 \%, 2.8 \pm 0.7 \%$ and $2.7 \pm$ $0.6 \%$ on average for the four isotopes, respectively. With combined rotated and shifted maps and altered attenuation coefficients, the average changes were $5.8 \pm 5.4 \%$, $4.4 \pm 3.2 \%, 3.3 \pm 2.2 \%$ and $3.9 \pm 2.2 \%$ for ${ }^{125} \mathrm{I},{ }^{201} \mathrm{Tl},{ }^{99 \mathrm{~m}} \mathrm{Tc}$ and ${ }^{111} \mathrm{In}$, respectively. The quantitative errors of the reference images, as described in [16], are listed in the first data column of Table 3 for reasons of comparison.

\section{Discussion}

We previously have shown that quantitative differences between uniform and non-uniform attenuation correction are quite small in micro-SPECT [16]. In this study, the influence of other attenuation map imperfections (i.e. misregistration and global errors in attenuation coefficient) on the quantitative accuracy of attenuationcorrected SPECT was investigated. For this purpose, we mimicked misregistration between micro-SPECT and micro-CT, and global deviation of attenuation coefficients. A mismatch of a few millimetres is already a big error for image registration in small-animal imaging: e.g. Ji et al. [31] showed that when using a proper calibration method for co-registration and a special bed mounting interface, the accuracy of small-animal SPECT-CT registration can reach sub-millimetre accuracy without using extra markers. Deviation of attenuation coefficients can be caused by the errors in CT voxel values (in $\mathrm{HU}$ ) or from the method that translates HU numbers into linear

Table 2 NRMSDs (in \%) between phantom images corrected with inaccurate maps and with accurate maps

\begin{tabular}{|c|c|c|c|c|c|c|c|c|}
\hline \multirow[t]{4}{*}{ Isotope } & \multicolumn{8}{|c|}{ Rotation, shift and $\times \mu$} \\
\hline & $0^{\circ}$ & $0^{\circ}$ & $0^{\circ}$ & $0^{\circ}$ & $15^{\circ}$ & $0^{\circ}$ & $0^{\circ}$ & $15^{\circ}$ \\
\hline & $1 \mathrm{~mm} X$ & $3 \mathrm{~mm} X$ & $1 \mathrm{~mm} Y$ & $3 \mathrm{~mm} Y$ & $0 \mathrm{~mm}$ & $0 \mathrm{~mm}$ & $0 \mathrm{~mm}$ & $3 \mathrm{~mm} X$ \\
\hline & $1.0 \mu$ & $1.0 \mu$ & $1.0 \mu$ & $1.0 \mu$ & $1.0 \mu$ & $0.9 \mu$ & $1.1 \mu$ & $1.1 \mu$ \\
\hline 225 & 1.7 & 4.9 & 1.7 & 4.7 & 0.8 & 4.4 & 4.6 & 6.3 \\
\hline${ }^{201} \mathrm{Tl}$ & 0.8 & 2.2 & 0.7 & 2.2 & 0.4 & 2.4 & 2.5 & 3.0 \\
\hline${ }^{99 \mathrm{~m} \mathrm{Tc}}$ & 0.6 & 1.8 & 0.6 & 1.8 & 0.4 & 2.1 & 2.1 & 2.5 \\
\hline${ }^{111} \ln$ & 0.6 & 1.7 & 0.6 & 1.6 & 0.3 & 1.9 & 2.0 & 2.3 \\
\hline
\end{tabular}



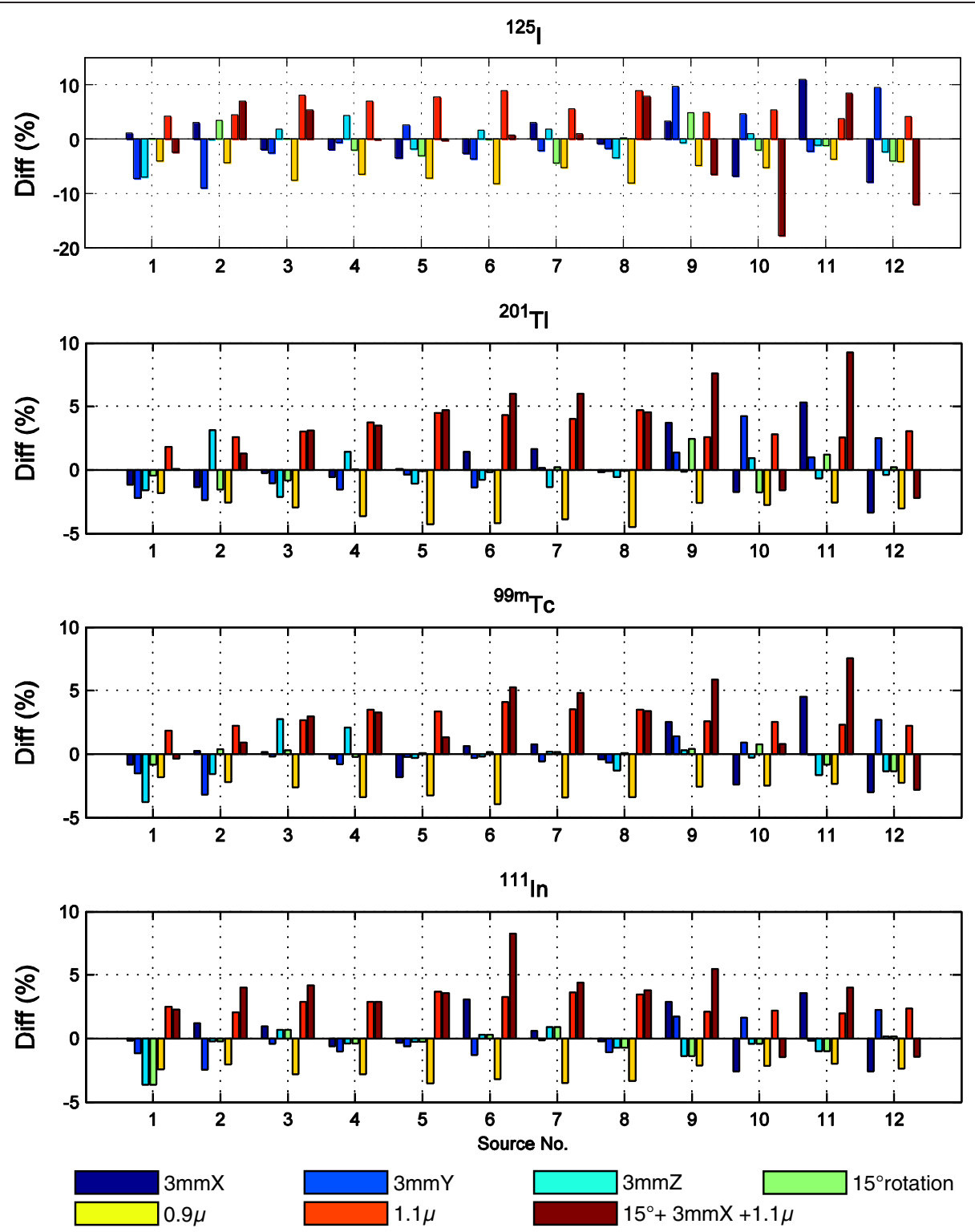

Figure 4 Differences between source activities in rat SPECT images corrected with inaccurate attenuation maps and with accurate maps. The differences are in percent (\%).

Table 3 Average absolute quantitative errors and average absolute changes of source activities

\begin{tabular}{|c|c|c|c|c|c|c|c|c|c|c|c|}
\hline \multirow[t]{4}{*}{ Isotope } & \multirow{4}{*}{$\begin{array}{l}\text { Quantitative } \\
\text { error }^{\mathrm{a}}\end{array}$} & \multicolumn{10}{|c|}{ Changes induced by map inaccuracies (rotation, shift and/or $\mu$ change) with respect to references } \\
\hline & & $0^{\circ}$ & $0^{\circ}$ & $0^{\circ}$ & $0^{\circ}$ & $0^{\circ}$ & $0^{\circ}$ & $15^{\circ}$ & $0^{\circ}$ & $0^{\circ}$ & $15^{\circ}$ \\
\hline & & $1 \mathrm{~mm} X$ & $3 \mathrm{~mm} X$ & $1 \mathrm{~mm} Y$ & $3 \mathrm{~mm} \mathrm{Y}$ & $1 \mathrm{~mm} \mathrm{Z}$ & $3 \mathrm{~mm} \mathrm{Z}$ & $0 \mathrm{~mm}$ & $0 \mathrm{~mm}$ & $0 \mathrm{~mm}$ & $3 \mathrm{~mm} X$ \\
\hline & & $1.0 \mu$ & $1.0 \mu$ & $1.0 \mu$ & $1.0 \mu$ & $1.0 \mu$ & $1.0 \mu$ & $1.0 \mu$ & $0.9 \mu$ & $1.1 \mu$ & $1.1 \mu$ \\
\hline 125 & $2.1 \pm 6.5$ & $1.5 \pm 1.1$ & $3.9 \pm 3.1$ & $1.6 \pm 1.2$ & $4.6 \pm 3.3$ & $0.9 \pm 0.9$ & $2.3 \pm 1.9$ & $2.1 \pm 1.8$ & $5.8 \pm 1.7$ & $6.1 \pm 1.9$ & $5.8 \pm 5.4$ \\
\hline${ }^{201} \mathrm{Tl}$ & $3.3 \pm 2.5$ & $0.6 \pm 0.6$ & $1.7 \pm 1.6$ & $0.6 \pm 0.4$ & $1.5 \pm 1.2$ & $0.5 \pm 0.5$ & $1.2 \pm 0.8$ & $0.8 \pm 0.8$ & $3.2 \pm 0.8$ & $3.3 \pm 0.9$ & $4.4 \pm 3.2$ \\
\hline${ }^{99 \mathrm{~m}} \mathrm{Tc}$ & $2.0 \pm 2.5$ & $0.6 \pm 0.5$ & $1.5 \pm 1.4$ & $0.4 \pm 0.4$ & $1.0 \pm 1.0$ & $0.5 \pm 0.4$ & $1.3 \pm 1.2$ & $0.5 \pm 0.4$ & $2.8 \pm 0.6$ & $2.9 \pm 0.7$ & $3.3 \pm 2.2$ \\
\hline${ }^{111} \ln$ & $2.0 \pm 2.7$ & $0.6 \pm 0.4$ & $1.6 \pm 1.3$ & $0.4 \pm 0.3$ & $1.2 \pm 0.8$ & $0.3 \pm 0.4$ & $0.8 \pm 1.0$ & $0.5 \pm 0.8$ & $2.7 \pm 0.6$ & $2.8 \pm 0.6$ & $3.9 \pm 2.2$ \\
\hline
\end{tabular}

Average absolute quantitative errors (in \%) of source activities in reference rat SPECT images were calculated with respect to dose calibrator measurements (1st data column), and average absolute changes (in \%) in source activities due to attenuation map inaccuracies were calculated with respect to reference images

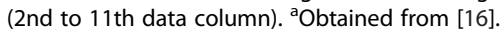


attenuation coefficients. The system error of CT (in HU) can be eliminated by re-calibration with a water phantom, and other effects such as ring artefacts and beamhardening effects can be well controlled and suppressed with proper reconstruction algorithms. Many reliable models for translating $\mathrm{HU}$ to attenuation coefficients for attenuation correction in SPECT and positron emission tomography have been proposed and evaluated, e.g. in $[32,33]$. Therefore, the level of the inaccuracies set in our present investigation is expected to exceed those applied in most real small-animal studies.
For both phantom and animal experiments, the changes in image quantitation due to strong attenuation map inaccuracies were less than about $5 \%$, except for ${ }^{125}$ I (worst case approximately $10 \%$ ), which can be attributed to ${ }^{125} \mathrm{I}$ attenuation that is more prominent.

In the phantom studies, quantitation changes due to attenuation map shifts were small inside the phantom, but increased close to the outer edge to a maximum of about $5 \%$ for ${ }^{125} \mathrm{I}$ and about $2 \%$ to $3 \%$ for ${ }^{201} \mathrm{Tl}$, ${ }^{99 \mathrm{~m}} \mathrm{Tc}$ and ${ }^{111}$ In. Although sharp attenuation and activity changes exist at the edges of the air chambers in the
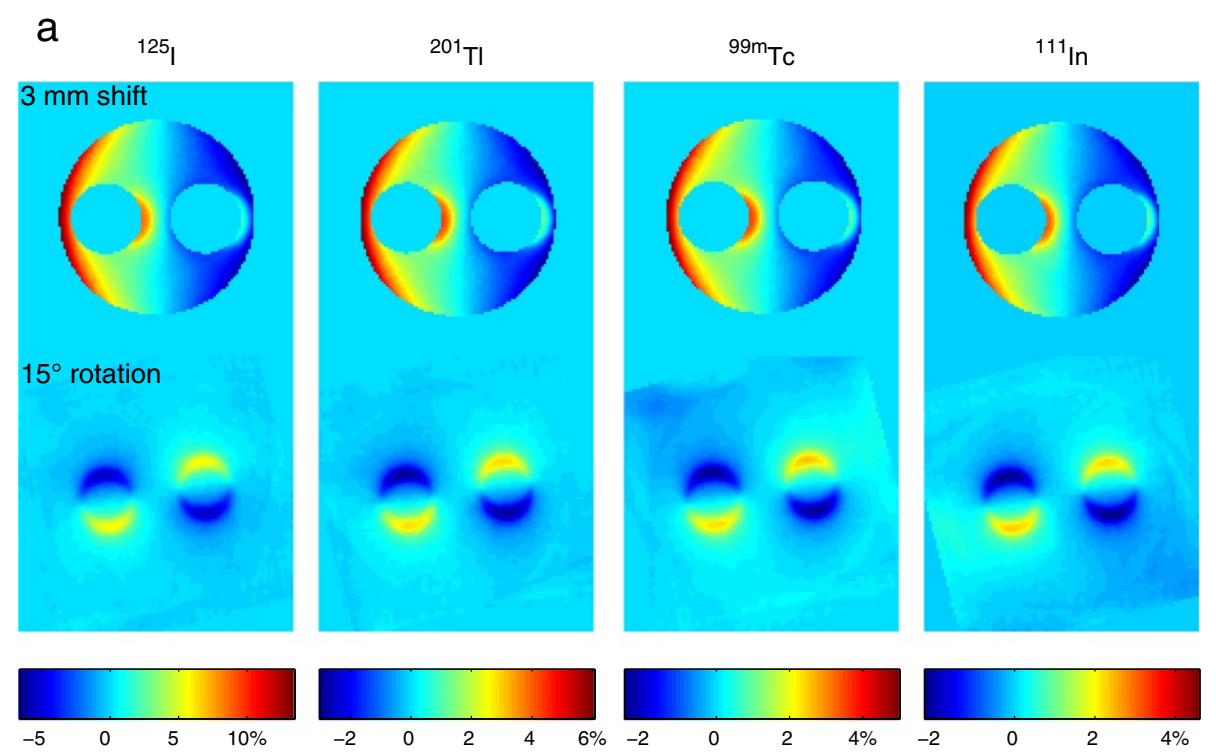

b
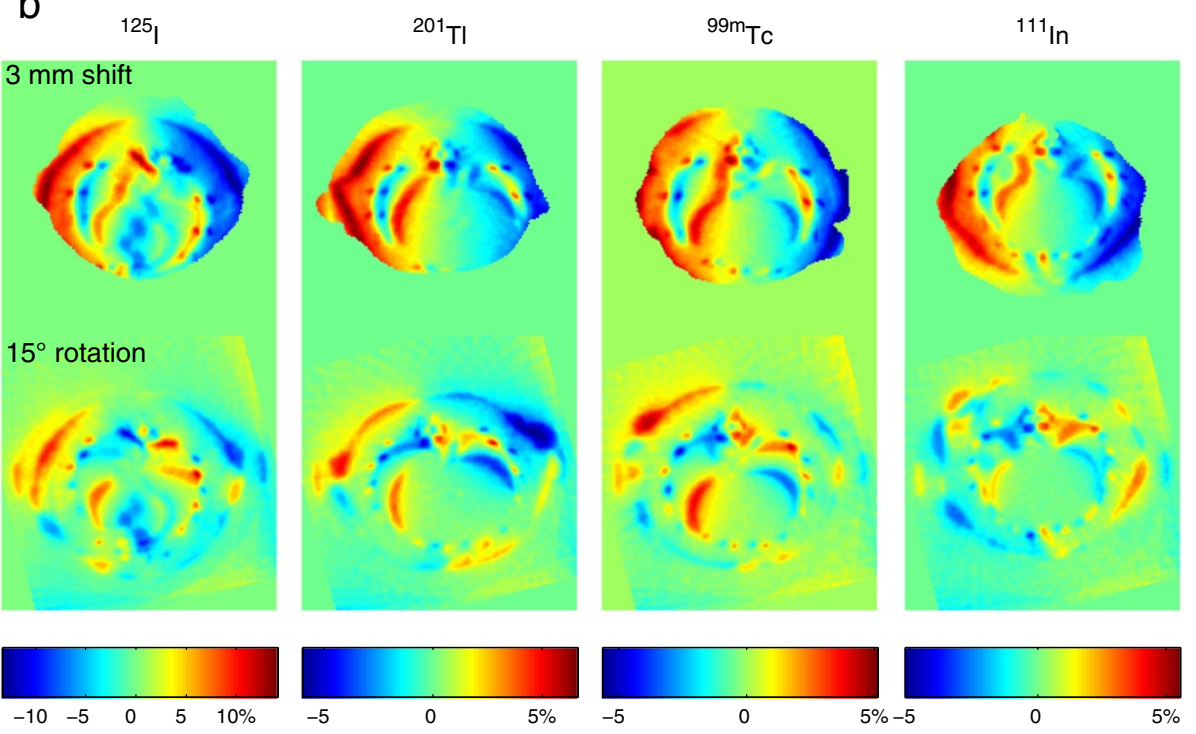

Figure 5 Relative differences in attenuation due to $3-\mathrm{mm}$ shifts or $15^{\circ}$ rotation. (a) Phantom heterogeneous slices and (b) rat thoracic slices. 
phantom, the changes in TF maps are rather smooth. As a result, quantification errors due to misregistration stayed also small in these regions. Quantitation changes caused by map rotation were smaller than the changes by shifts, and because the axis of rotation was closely aligned with the axis of the phantom, the changes only existed close to the air chambers. This may be relevant for quantification in e.g. tumours that are close to lungs or in cardiac imaging. These changes of measured activity concentration were consistent with the relative differences between the reference TF maps and the shifted or rotated maps, as shown in Figure 5a. When imaging the small sources in the rat cadavers, the activity changes in a subset of sources due to shifts and rotation were a bit larger than changes in the phantom, which can be explained by the effects of non-uniform attenuation close to the sources. Again, the influence of rotation on map accuracy was smaller than that of shifts, as shown in the rat thoracic slices in Figure 5b. Since both large shifts and rotation have limited effects on quantitative accuracy in our emulation, we believe that the typical misalignments between micro-SPECT and micro-CT caused by
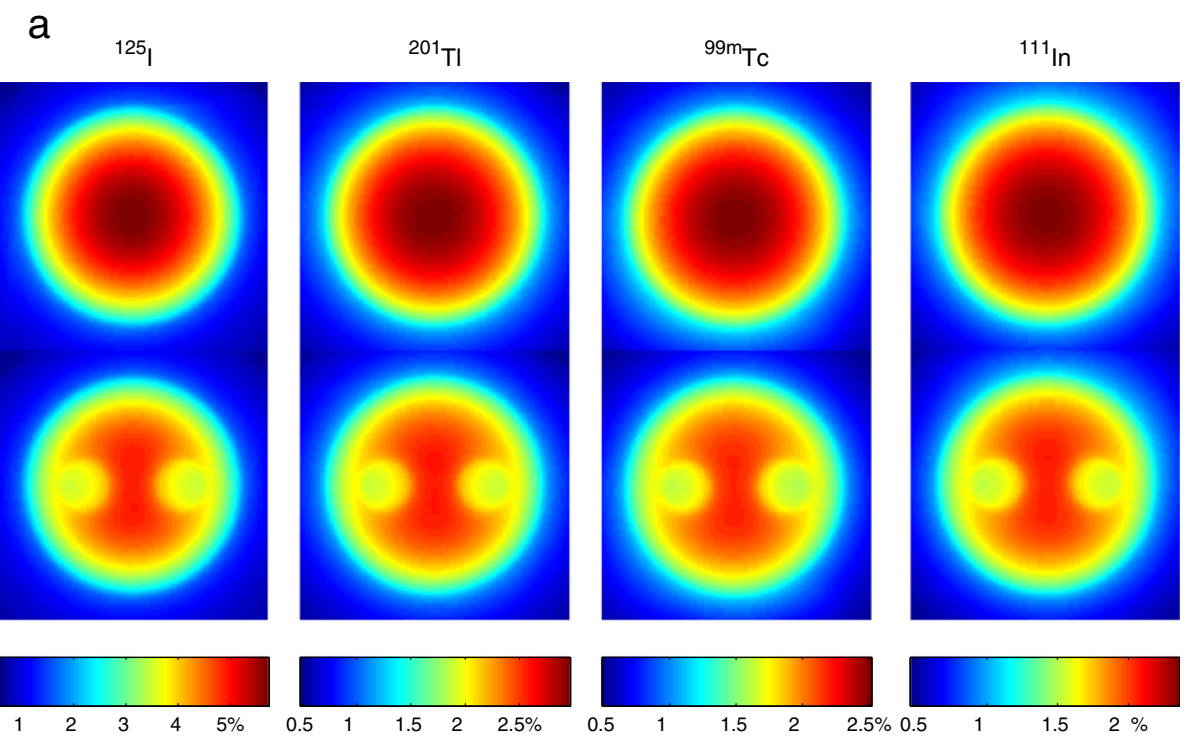

b
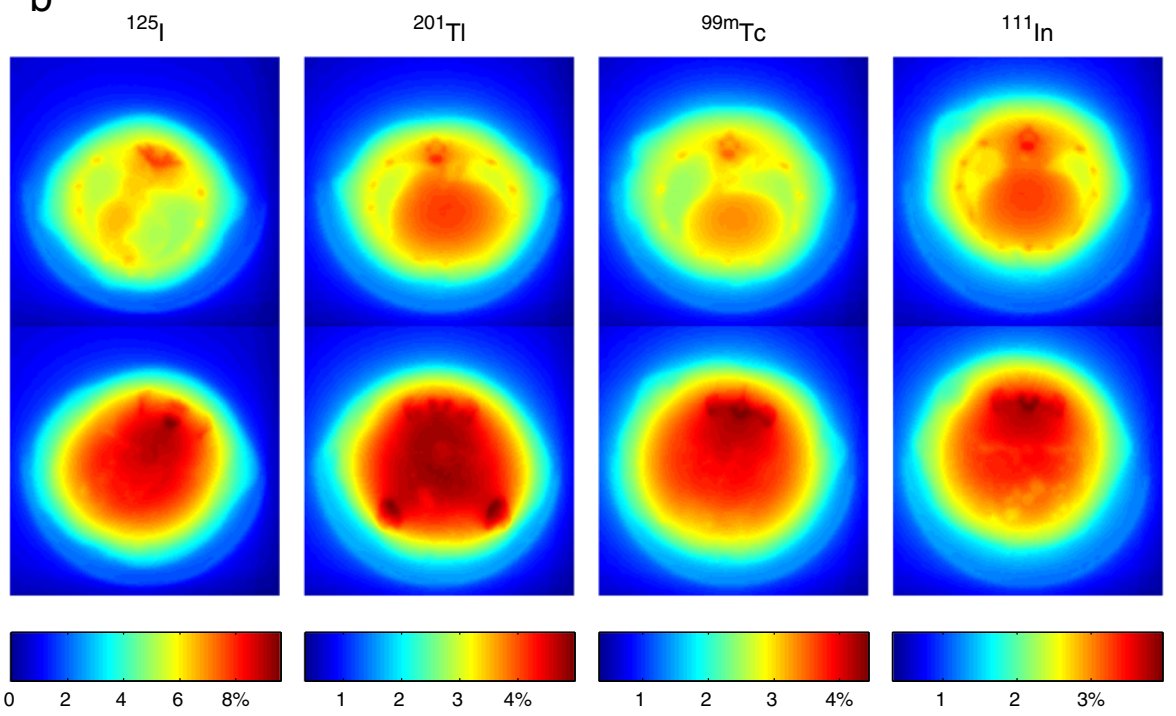

Figure 6 Relative differences in attenuation due to multiplying attenuation coefficients by 1.1. (a) Two different phantom slices and (b) two different slices through each rat. 
sub-optimal registration and/or animal movement during scans will not often have a prominent impact to SPECT quantitative accuracy.

With attenuation coefficients globally altered by $\pm 10 \%$, similar global over- or underestimation was observed in both phantom and animal studies. We also examined the relative differences between the reference TF maps and the ones derived from 10\% increased attenuation coefficients. Figure $6 \mathrm{a}$ shows the differences in two transaxial slices of phantom TF maps for each isotope. One slice was from the part of the phantom without air chambers, and the other one was from the part that contains the two air chambers. Figure $6 \mathrm{~b}$ shows the differences in two slices through the rat TF maps: one slice across the thorax and the other across the abdomen of each rat. It is clear that with a global change of the attenuation coefficients, the changes in the TF maps are not homogenous. The effects are larger around bone areas as shown in Figure $6 \mathrm{~b}$ and are smaller at areas with low density such as the air chambers in Figure 6a and lungs in Figure 6b. Ostensibly, this implies that the attenuation coefficient accuracy in and around the bones may play a relatively important role in the accuracy of SPECT attenuation correction. However, the contribution of bone attenuation is limited in the integral in Equation 1 for small animals so that effects on quantification would not spread widely, as shown in the 'Results' section and in Figure 6b.

Without attenuation correction, activities were roughly underestimated by $40 \%$ for ${ }^{125} \mathrm{I}$ and $20 \%$ for ${ }^{201} \mathrm{Tl},{ }^{99 \mathrm{~m}} \mathrm{Tc}$ and ${ }^{111} \mathrm{In}$. Even the largest inaccuracies in attenuation maps investigated here effectively took away only one eighth of the benefit from the attenuation correction. Since accuracy of the attenuation coefficients and alignment between micro-SPECT and micro-CT in practice are usually better than in the worst situations emulated in the present study, effects of attenuation map inaccuracies on micro-SPECT quantification will usually be small.

\section{Conclusions}

For the more commonly used SPECT isotopes like ${ }^{201} \mathrm{Tl}$, ${ }^{99 \mathrm{~m}} \mathrm{Tc}$ and ${ }^{111} \mathrm{In}$, misalignments up to a few millimetres or in the order of $10^{\circ}$ to $15^{\circ}$ between micro-SPECT and micro-CT, and/or global attenuation coefficient inaccuracies in the range of $\pm 10 \%$ have quite small effects on micro-SPECT quantification. Therefore, we conclude that micro-SPECT quantification is quite robust to imperfections in attenuation maps for most applications.

\section{Abbreviations}

CT: Computed tomography; NRMSD: Normalized root mean square deviation; SPECT: Single-photon emission computed tomography; TF: Transmitted fraction; VOI: Volume of interest.

\section{Competing interests}

Freek J. Beekman is a stockholder of and gets honoraria and grant/research support from MILabs. Hugo A. Gratama van Andel is an employee of MILabs. The other authors declare that they have no competing interests.

\section{Authors' contributions}

CW designed the study, performed the experiments, analysed and interpreted the data, and drafted and revised the manuscript. HAGvA analysed and interpreted the data and revised the manuscript. PL COperformed the experiments and revised the manuscript. OCB participated in the study design and coordination and revised the manuscript. FJB conceived of and designed the study, participated in its coordination and drafted and revised the manuscript. All authors read and approved the final manuscript.

\section{Acknowledgments}

This research was partly performed within the framework of CTMM, the Center for Translational Molecular Medicine (www.ctmm.nl), project EMINENCE (grant 01C-204). We are grateful to Bianca Lemmers-de Weem (Central Animal Facility, Radboud University Nijmegen, Nijmegen, the Netherlands) for technical assistance and Pieter E. B. Vaissier (Section Radiation, Detection \& Medical Imaging, Delft University of Technology, Delft, the Netherlands) for suggestions and comments on the manuscript.

\section{Author details}

${ }^{1}$ Section Radiation, Detection \& Medical Imaging, Delft University of Technology, Mekelweg 15, Delft 2629 JB, the Netherlands. ${ }^{2}$ Rudolf Magnus Institute of Neuroscience, University Medical Center Utrecht, STR 5.101, Univeriteitsweg 100, Utrecht 3584 CG, the Netherlands. ${ }^{3}$ MILabs B.V., Heidelberglaan 100, Utrecht 3584 CX, the Netherlands. ${ }^{4}$ Department of Nuclear Medicine, Radboud University Nijmegen Medical Centre, Geert Grooteplein 8, Nijmegen 6525 GA, the Netherlands.

Received: 10 December 2012 Accepted: 23 January 2013 Published: 31 January 2013

\section{References}

1. Miao Y, Benwell K, Quinn TP: ${ }^{99 \mathrm{~m}} \mathrm{Tc}$ - and ${ }^{111} \mathrm{In}$-labeled alphamelanocyte-stimulating hormone peptides as imaging probes for primary and pulmonary metastatic melanoma detection. J NuCl Med 2007, 48:73-80.

2. de Visser M, Bernard HF, Erion JL, Schmidt MA, Srinivasan A, Waser B, Reubi $J C$, Krenning EP, de Jong M: Novel ${ }^{111}$ In-labelled bombesin analogues for molecular imaging of prostate tumours. Eur I Nucl Med Mol Imaging 2007, 34:1228-1238

3. Zhang J, Nie L, Razavian M, Ahmed M, Dobrucki LW, Asadi A, Edwards DS, Azure M, Sinusas AJ, Sadeghi MM: Molecular imaging of activated matrix metalloproteinases in vascular remodeling. Circulation 2008, 118:1953-1960

4. Hoang B, Lee H, Reilly RM, Allen C: Noninvasive monitoring of the fate of ${ }^{111}$ In-labeled block copolymer micelles by high resolution and high sensitivity microSPECT/CT imaging. Mol Pharm 2009, 6:581-592.

5. Golestani R, Wu C, Tio RA, Zeebregts CJ, Petrov AD, Beekman FJ, Dierckx RA, Boersma HH, Slart RH: Small-animal SPECT and SPECT/CT: application in cardiovascular research. Eur J Nucl Med Mol Imaging 2010, 37:1766-1777.

6. Kagadis GC, Loudos G, Katsanos K, Langer SG, Nikiforidis GC: In vivo small animal imaging: current status and future prospects. Med Phys 2010, 37:6421-6442.

7. Vaneycken I, Devoogdt N, Van Gassen N, Vincke C, Xavier C, Wernery U, Muyldermans S, Lahoutte T, Caveliers V: Preclinical screening of anti-HER2 nanobodies for molecular imaging of breast cancer. FASEB J 2011, 25:2433-2446

8. Vegt E, Melis M, Eek A, de Visser M, Brom M, Oyen WJG, Gotthardt M, de Jong M, Boerman OC: Renal uptake of different radiolabelled peptides is mediated by megalin: SPECT and biodistribution studies in megalindeficient mice. Eur J Nucl Med Mol Imaging 2011, 38:623-632.

9. Umeda IO, Tani K, Tsuda K, Kobayashi M, Ogata M, Kimura S, Yoshimoto M, Kojima S, Moribe K, Yamamoto K, Moriyama N, Fujii H: High resolution SPECT imaging for visualization of intratumoral heterogeneity using a SPECT/CT scanner dedicated for small animal imaging. Ann Nucl Med 2012, 26:67-76. 
10. Hwang AB, Taylor CC, VanBrocklin HF, Dae MW, Hasegawa BH: Attenuation correction of small animal SPECT images acquired with I-125iodorotenone. IEEE Trans NuCl Sci 2006, 53:1213-1220.

11. Vanhove C, Defrise M, Bossuyt A, Lahoutte T: Improved quantification in single-pinhole and multiple-pinhole SPECT using micro-CT information. Eur J Nucl Med Mol Imaging 2009, 36:1049-1063.

12. Gullberg GT, Huesman RH, Malko JA, Pelc NJ, Budinger TF: An attenuated projector-backprojector for iterative SPECT reconstruction. Phys Med Biol 1985, 30:799-816.

13. Ogawa $K$, Harata $Y$, Ichihara T, Kubo A, Hashimoto S: A practical method for position-dependent Compton-scatter correction in single photon emission CT. IEEE Trans Med Imaging 1991, 10:408-412.

14. Chang LT: A method for attenuation correction in radionuclide computed tomography. IEEE Trans NuCl Sci 1978, 25:638-643.

15. Wu C, van der Have F, Vastenhouw B, Dierckx RA, Paans AM, Beekman FJ: Absolute quantitative total-body small-animal SPECT with focusing pinholes. Eur J Nucl Med Mol Imaging 2010, 37:2127-2135.

16. Wu C, de Jong JR, van Andel HAG, van der Have F, Vastenhouw B, Laverman P, Boerman OC, Dierckx RA, Beekman FJ: Quantitative multipinhole small-animal SPECT: uniform versus non-uniform Chang attenuation correction. Phys Med Biol 2011, 56:N183-N193.

17. Tonge CM, Manoharan M, Lawson RS, Shields RA, Prescott MC: Attenuation correction of myocardial SPECT studies using low resolution computed tomography images. Nucl Med Commun 2005, 26:231-237.

18. Tonge CM, Ellul G, Pandit M, Lawson RS, Shields RA, Arumugam P, Prescott $M C$ : The value of registration correction in the attenuation correction of myocardial SPECT studies using low resolution computed tomography images. Nucl Med Commun 2006, 27:843-852.

19. Goetze S, Brown TL, Lavely WC, Zhang Z, Bengel FM: Attenuation correction in myocardial perfusion SPECT/CT: effects of misregistration and value of reregistration. J Nucl Med 2007, 48:1090-1095.

20. Kennedy JA, Israel $O$, Frenkel A: Directions and magnitudes of misregistration of $\mathrm{CT}$ attenuation-corrected myocardial perfusion studies: incidence, impact on image quality, and guidance for reregistration. J Nucl Med 2009, 50:1471-1478.

21. Tsui BM, Kraitchman DL: Recent advances in small-animal cardiovascular imaging. J NuCl Med 2009, 50:667-670.

22. van der Have F, Vastenhouw B, Ramakers RM, Branderhorst W, Krah JO, Ji C, Staelens SG, Beekman FJ: U-SPECT-II: an ultra-high-resolution device for molecular small-animal imaging. J Nucl Med 2009, 50:599-605.

23. Wyckhuys T, Staelens S, Van Nieuwenhuyse B, Deleye S, Hallez H, Vonck K, Raedt R, Wadman W, Boon P: Hippocampal deep brain stimulation induces decreased rCBF in the hippocampal formation of the rat. Neuroimage 2010, 52:55-61.

24. Branderhorst W, Vastenhouw B, van der Have F, Blezer EL, Bleeker WK Beekman FJ: Targeted multi-pinhole SPECT. Eur J Nucl Med Mol Imaging 2011, 38:552-561.

25. Visser EP, Harteveld AA, Meeuwis APW, Disselhorst JA, Beekman FJ, Oyen WJG, Boerman OC: Image quality phantom and parameters for high spatial resolution small-animal SPECT. Nucl Instrum Meth A 2011 654:539-545.

26. Vastenhouw B, Beekman F: Submillimeter total-body murine imaging with U-SPECT-I. J NuCl Med 2007, 48:487-493.

27. Branderhorst W, Vastenhouw B, Beekman FJ: Pixel-based subsets for rapid multi-pinhole SPECT reconstruction. Phys Med Biol 2010, 55:2023-2034

28. van der Have F, Vastenhouw B, Rentmeester M, Beekman FJ: System calibration and statistical image reconstruction for ultra-high resolution stationary pinhole SPECT. IEEE Trans Med Imaging 2008, 27:960-971.

29. LaCroix KJ, Tsui BMW, Hasegawa BH, Brown JK: Investigation of the use of $\mathrm{X}$-ray CT images for attenuation compensation in SPECT. IEEE Trans NuCl Sci 1994, 41:2793-2799.

30. Chantler CT: Detailed tabulation of atomic form factors, photoelectric absorption and scattering cross section, and mass attenuation coefficients in the vicinity of absorption edges in the soft $X$-ray ( $Z=30-36, Z=60-89, E=0.1-10 \mathrm{keV}$ )-addressing convergence issues of earlier work. J Synchrotron Radiat 2001, 8:1124.

31. Ji C, van der Have F, Gratama van Andel H, Ramakers R, Beekman F: Accurate coregistration between ultra-high-resolution micro-SPECT and circular cone-beam micro-CT scanners. Int J Biomed Imaging 2010, 2010:654506.
32. Brown S, Bailey DL, Willowson K, Baldock C: Investigation of the relationship between linear attenuation coefficients and $\mathrm{CT}$ Hounsfield units using radionuclides for SPECT. Appl Radiat Isotopes 2008, 66:1206-1212.

33. Ay MR, Shirmohammad M, Sarkar S, Rahmim A, Zaidi H: Comparative assessment of energy-mapping approaches in CT-based attenuation correction for PET. Mol Imaging Biol 2011, 13:187-198.

doi:10.1186/2191-219X-3-7

Cite this article as: Wu et al: Effects of attenuation map accuracy on attenuation-corrected micro-SPECT images. EJNMMI Research 2013 3:7.

\section{Submit your manuscript to a SpringerOpen ${ }^{\odot}$ journal and benefit from:}

- Convenient online submission

- Rigorous peer review

- Immediate publication on acceptance

- Open access: articles freely available online

- High visibility within the field

- Retaining the copyright to your article

Submit your next manuscript at $>$ springeropen.com 